\title{
Effect of adjuvant sleep hygiene psychoeducation and lorazepam on depression and sleep quality in patients with major depressive disorders: results from a randomized three-arm intervention
}

\author{
This article was published in the following Dove Press journal: \\ Neuropsychiatric Disease and Treatment \\ 22 June 2016 \\ Number of times this article has been viewed
}

\begin{abstract}
Alireza Rahimi'
Mohammad Ahmadpanah'

Farshid Shamsaei'

Fatemeh Cheraghi

Dena Sadeghi Bahmani ${ }^{3}$

Edith Holsboer-Trachsler ${ }^{3}$

Serge Brand ${ }^{3,4}$

'Behavioral Disorders and Substances Abuses Research Center, Hamadan University of Medial Sciences, Hamadan, ${ }^{2}$ Research Center for Chronic Disease Care at Home, Hamadan University of Medial Sciences, Hamadan, Iran; ${ }^{3}$ Psychiatric Clinics of the University of Basel, Center for Affective, Stress and Sleep Disorders (ZASS), ${ }^{4}$ Department of Sport, Exercise, and Health, Division of Sport and Psychosocial Health, University of Basel, Basel, Switzerland
\end{abstract}

Background: Sleep disturbances are a common co-occurring disturbance in patients with major depressive disorders (MDDs) and accordingly deserve particular attention. Using a randomized design, we investigated the effects of three different adjuvant interventions on sleep and depression among patients with MDD: a sleep hygiene program (SHP), lorazepam (LOR), and their combination (SHP-LOR).

Methods: A total of 120 outpatients with diagnosed MDD (mean age: 48.25 years; $56.7 \%$ females) and treated with a standard SSRI (citalopram at $20-40 \mathrm{mg}$ at therapeutic level) were randomly assigned to one of the following three conditions: SHP ( $n=40), \operatorname{LOR}(1 \mathrm{mg} / \mathrm{d} ; \mathrm{n}=40)$, SHP-LOR ( $1 \mathrm{mg} / \mathrm{d} ; \mathrm{n}=40)$. At the beginning and at the end of the study 8 weeks later, patients completed two questionnaires, the Pittsburgh Sleep Quality Index to assess sleep and the Beck Depression Inventory to assess symptoms of depression.

Results: Sleep disturbances decreased over time and in all groups. No group differences or interactions were observed. Symptoms of depression decreased over time and in all three groups. Reduction in symptoms of depression was greatest in the SHP-LOR group and lowest in the LOR group.

Conclusion: The pattern of results suggests that all three adjuvant treatments improved symptoms of sleep disturbances and depression, with greater benefits for the SHP-LOR for symptoms of depression, but not for sleep. Nevertheless, risks and benefits of benzodiazepine prescriptions should be taken into account.

Keywords: sleep hygiene, psychoeducation, pharmacotherapy, lorazepam, sleep disturbances, depression

\section{Introduction}

Restoring sleep is associated with a broad variety of physical, ${ }^{1}$ emotional, ${ }^{2,3}$ cognitive, ${ }^{4,5}$ and social benefits. ${ }^{6}$ Poor and nonrestoring sleep, in contrast, leads to impairments on physiological, emotional, cognitive, and social levels. In other words, poor sleep is associated with impaired daily functioning. This observation holds particularly true for people with mental disorders; sleep disturbances are nearly universal features of psychiatric disorders, especially mood disorders. Indeed, research investigating associations between sleep and affective illness has largely focused on depression and major depressive disorder (MDD). ${ }^{7-10}$

In this regard, epidemiological data indicate that people with psychiatric disorders account for $30 \%-40 \%$ of those in the community reporting symptoms of insomnia. ${ }^{11}$
Correspondence: Serge Brand Center for Affective, Stress and Sleep Disorders, Psychiatric Clinics of the University of Basel, Wilhelm Klein-Strasse 27, 40I2 Basel, Switzerland Tel +4 I 613255 II 4

Fax $+4 \mid 613255513$

Email serge.brand@upkbs.ch 
Of those individuals with insomnia, 40\%-60\% display features of depression. Likewise, disturbances in sleep and wakefulness, such as insomnia, excessive sleepiness, and fatigue, are experienced by most individuals suffering from depression, and sleep disorder is among the most prevalent symptoms of depression, with rates of $\sim 80 \%{ }^{12,13}$ As regards the direction of influence (whether sleep disturbances lead to depressive symptoms, or depressive symptoms lead to sleep disturbances), there is growing evidence that sleep disturbances are at the beginning of increasing symptoms of depression. In their meta-analysis, Lovato and Gradisar ${ }^{14}$ showed that sleep disturbances preceded symptoms of depression among adolescents. In adults, a history of persistent insomnia has been associated with a significantly increased risk of developing a new episode of depression and bipolar disorder. ${ }^{15}$ Likewise, poor objective sleep has predicted the recurrence of a further depressive episode, ${ }^{10,16,17}$ and it is estimated that poor sleep precedes an episode of depression in $40 \%$ of cases. ${ }^{9}$ Further, there is strong evidence that preexisting insomnia contributes greatly to the course and severity of depressive disorders and even predicts relapse or poor outcome. ${ }^{18}$ In addition and most dramatically, poor sleep increases the risk of suicidal ideation ${ }^{19-21}$ and also the likelihood of a poorer response to combined pharmacological and psychological treatments of depression. ${ }^{17}$

There have typically been three options for the treatment of sleep disturbances in general and in patients with MDD: psychopharmacotherapy, ${ }^{22-25}$ psychotherapy/ psychoeducation, ${ }^{26-28}$ and the combination of psychopharmacotherapy and psychotherapy. ${ }^{29-33}$ Riemann and Perlis ${ }^{30}$ have argued the need for further research on the option of combining psychopharmacotherapy and psychotherapy. We took this observation into account and compared all three possibilities as adjuvant treatments of MDD with a standard SSRI in a randomized three-arm study. Specifically, patients with MDDs and sleep disturbances were randomly assigned to one of the following conditions: adjuvant sleep hygiene program (SHP), adjuvant psychopharmacotherapy (lorazepam [LOR], $1 \mathrm{mg} / \mathrm{d}$ ), and adjuvant sleep hygiene program combined with adjuvant psychopharmacotherapy (lorazepam [SHP-LOR], $1 \mathrm{mg} / \mathrm{d}$ ). In this way, we expected to find some answers as regards to the treatment options for sleep disturbances in patients with MDD.

The following four hypotheses were formulated. First, following previous findings, ${ }^{34-37}$ we expected an improvement in symptoms of depression over time as an effect of the standard treatment with an antidepressant, irrespective of the adjuvant treatment. Second, in light of previous findings, ${ }^{26-28}$ we expected a more beneficial effect of the adjuvant SHP on sleep, compared with the other two treatment options (SHP-LOR and LOR). Third, again following previous findings, ${ }^{29-33}$ we expected a more beneficial effect of the adjuvant combined treatment (SHP-LOR) on depression, compared with the other two treatment methods (SHP and LOR). Fourth, on the basis of previous findings, ${ }^{10,38-41}$ we anticipated that symptoms of depression and sleep disturbances would be associated both at the beginning and at the end of the study.

\section{Methods}

Procedure

Eligible outpatients were approached and recruited between September 2013 and April 2014 at the outpatient's center of the Farshchian Psychiatry Hospital of Hamedan (Iran). Of the 245 patients approached, $129(52.65 \%)$ met the inclusion and exclusion criteria ("Sample" section) and agreed to participate; 120 completed the study (statistical data analysis was made per protocol, and the CONSORT flow diagram [Figure 1] shows sampling and participants' assignments). Participants were fully informed about the aims of the 8-week study and the voluntary basis of their participation. Further, patients were assured of the anonymous nature of the data gathering. Next, they signed a written informed consent. Thereafter, psychopharmacological treatment of MDD was initiated. Two to three weeks before the study commenced, patients began treatment with citalopram, a standard SSRI, at therapeutic levels (20-40 mg/d), which was kept constant throughout the entire study. Psychiatrists and clinical psychologists not otherwise involved in the study performed a clinical assessment, including a diagnostic interview ("Sample" section). Patients completed questionnaires related to sleep disturbances and to depression ("Tools" section) both at the beginning and at the end of the study. The Ethical Committee of the Hamadan University of Medical Sciences approved the study, which was executed according to the ethical standards laid down in the Declaration of Helsinki. Further, the study was registered in Iranian Registry of Clinical Trials (IRCT number: 2012100411004N1).

\section{Sample}

Outpatients with diagnosed MDD were enrolled in the study. The inclusion criteria were 1) diagnosis of MDDs without psychotic features, according to the Diagnostic and Statistical Manual of Mental Disorders, Fifth Edition; 2 ) reported sleep disturbances; 3 ) aged between 18 years and 65 years; and 4) willing and able to follow the study protocol 


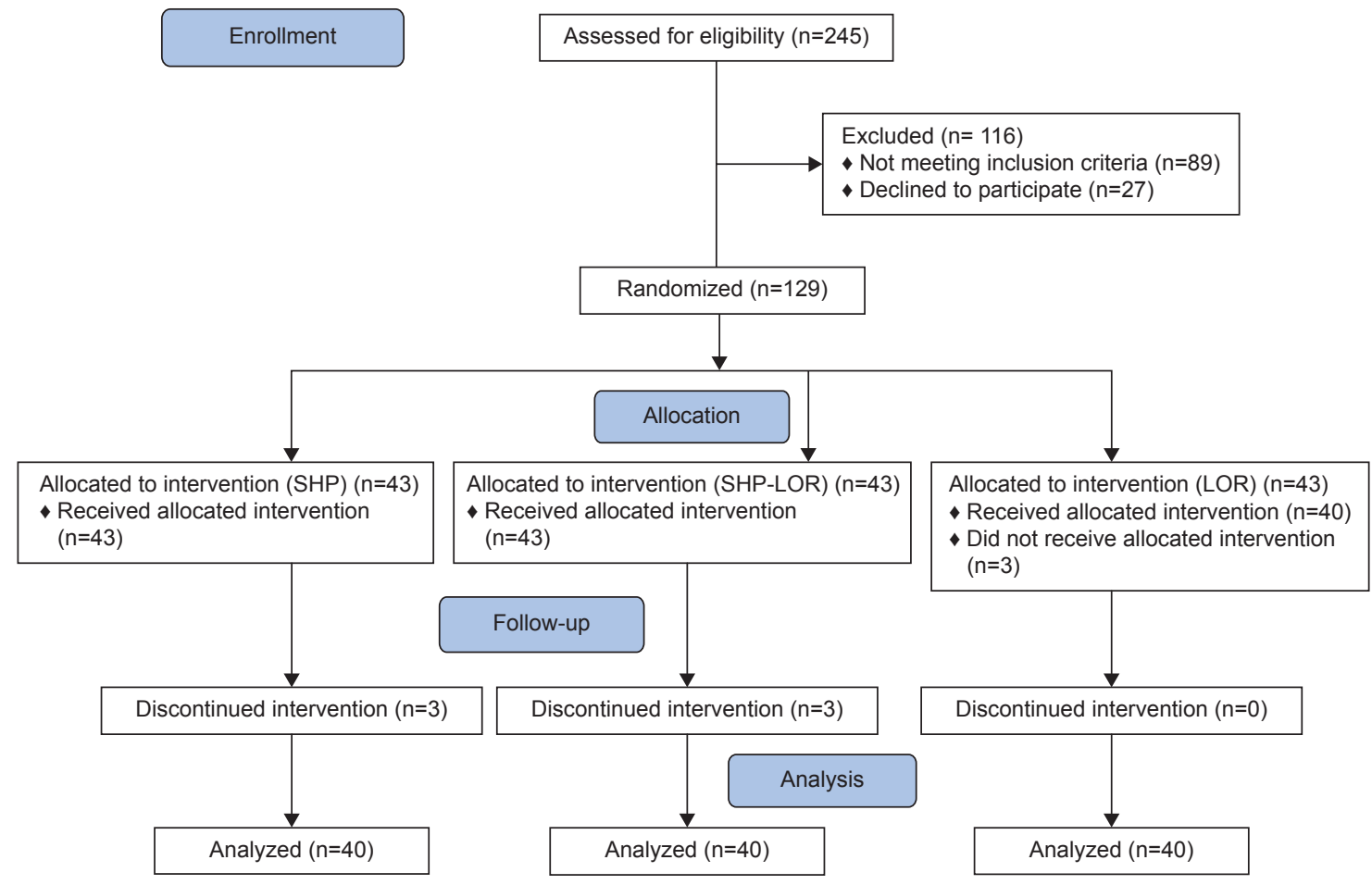

Figure I CONSORT 2010 flow diagram.

Abbreviations: SHP, sleep hygiene program; SHP-LOR, sleep hygiene program + lorazepam (Img/d); LOR, lorazepam (I mg/d).

and to complete the questionnaires. Exclusion criteria were 1) not meeting the inclusion criteria; 2) presence of a progressive illness (eg, cancer and dementia) directly related to the onset and course of insomnia; 3 ) use of medications with sleep-altering effects (eg, steroids); 4) lifetime diagnosis of any psychotic or bipolar disorder; 5) alcohol or drug abuse within the past year; 6) sleep apnea or restless legs ("Sleep-related assessment" section); and 7) night-shift work or irregular sleep pattern. All patients were treated with a standard SSRI, that is, with citalopram at a therapeutic level $(20-40 \mathrm{mg} / \mathrm{d})$.

\section{Psychiatric assessment}

Experts not further involved in the study performed the psychiatric assessment with the Mini International Neuropsychiatric Interview ${ }^{42}$ to allow selection of patients according to the inclusion and exclusion criteria.

\section{Sleep-related assessment}

First, experts asked about current sleep quality based on the Insomnia Severity Index..$^{43}$ Further, to exclude sleep disturbances due to sleep apnea and restless legs, the following questions were asked: sleep apnea: "Are you regularly snoring loudly during the night?" and "Does your partner [or somebody else] tell you that you stop breathing during the night?"; restless legs syndrome: "Do you feel discomfort in your legs in the evening such as an itch you can't scratch, a 'buzzing sensation', an unpleasant 'tickle that won't stop', a 'crawling'?" and "Do feelings of discomfort disappear when you start moving your legs?". Answering "yes" to one of the abovementioned questions led to exclusion from the study.

\section{Randomization and intervention groups}

A psychologist not further involved in the study prepared a total of 129 raffle tickets, 43 in each of three different colors, put them in a ballot box and stirred. At the start of the study, patients drew a raffle ticket and were assigned the group with the defined color.

The following groups and interventions were defined: 1) SHP ( $n=43), 2)$ tablet $1 \mathrm{mg}$ of LOR nightly ( $\mathrm{n}=43$ ), and 3) SHP-LOR nightly $(n=43)$.

\section{Interventions}

\section{Sleep hygiene program}

In addition to the standard treatment with citalopram, patients received four sessions (30-45 minutes; group or individual sessions) of a SHP, based on Silberman's Insomnia workbook. ${ }^{44}$ Psychologists not further involved in the study delivered these sessions. The first session took place at the beginning of the study, and the last session 2 weeks before the end of the study. Specifically, participants learned 
about basics of sleep hygiene such as keeping a regular sleep-wake schedule; avoiding abundant, late, and fatty dinners, but eating small, light, and easily digested meals to avoid being hungry during the night; moving a lot during the day and even in the evening; ${ }^{45}$ avoiding naps; keeping a sleep and activity log for seven consecutive days; avoiding stimulants in the evening (coffee, tea, coke, high sugar soft drinks, alcohol, drugs, and nicotine); going to bed later and getting up early to increase sleep need; and using the bedroom exclusively for romance and sleep (thus, avoiding screen time and eating in the bed room/bed). Further, during the sessions, participants were instructed in relaxation techniques to improve their ability to relax body and mind in the evening and so fall asleep more easily. During the four sessions, these techniques were practiced. Finally, patients also received a leaflet comprising information regarding sleep hygiene, the importance of sleep, and sleep hygiene practices. It contained representative information regarding adult's sleep and healthy sleep practices in general, as well as recommendations about sleep duration for adults, recommendations about the temporal organization of sleep, information about different behaviors and environmental factors that can influence sleep quality, and about the importance of sleep and consequences of sleepiness. The leaflet also contained an example of a sleep-wake diary and an explanation for its use. It was designed by professional graphic designers on a single unfolded sheet of paper, printed on both sides. The dimensions of the leaflet were $21 \times 21 \mathrm{~cm}^{2}$.

\section{Pharmacotherapy}

In addition to the standard treatment with citalopram, patients in the pharmacotherapy group were treated for 8 weeks with LOR. LOR is a benzodiazepine. Although the manufacturer advises discontinuing regular LOR intake after 3-4 weeks, the US Federal Drug and Food Administration has approved all hypnotics since 2005 without restriction as to duration of use. ${ }^{23}$ The focus of the four pharmacotherapy sessions (30-45 minutes) was on medication management; this involved education about medications, adjustment of dosage and dosage schedules, and discussions of adverse effects. All patients in the pharmacotherapy conditions began treatment with LOR at $1 \mathrm{mg} / \mathrm{d}$.

\section{Combined sleep hygiene program and pharmacotherapy}

In addition to the standard treatment with citalopram, patients assigned to this study condition had four sessions (45-50 minutes) each on sleep hygiene and on medication management, as described in the "Sleep hygiene program" and "Pharmacotherapy" sections. Thus, participants in this treatment condition received both the SHP and the medication (LOR).

\section{Tools}

Assessment of sleep patterns and quality (self-rating) To assess sleep patterns and quality, the Persian ${ }^{46}$ version of Pittsburgh Sleep Quality Index (PSQI) ${ }^{47}$ was employed. The PSQI is a self-report questionnaire that assesses sleep quality over a 1-month interval. It consists of 19 individual items generating seven "component" scores: subjective sleep quality, sleep latency (ie, how long it takes to fall asleep), sleep duration, habitual sleep efficiency (ie, the percentage of time in bed that one is asleep), sleep disturbances, use of sleep medication, and daytime dysfunction. Nazifi et $\mathrm{al}^{46}$ have reported appropriate internal consistency (Cronbach's alpha of 0.85 ) for its clinically calculated seven components. ${ }^{48,49}$ Nazifi et al's findings are consistent with those for the English version of the PSQI. ${ }^{47-49}$ Scores are combined according to the scoring criteria included with the form to produce a Global PSQI Score. Scores above 5 indicate clinically relevant levels of disturbed sleep.

\section{Assessment of symptoms of depression (self-rating)}

The Persian version of Beck Depression Inventory $\left(\mathrm{BDI}^{50}\right)$ was used to measure depressive symptoms. ${ }^{51}$ The BDI samples self-reported symptoms of depression. The questionnaire consists of 21 items and asks about different dimensions such as depressive mood, loss of appetite, sleep disorders, suicidality, and similar issues. Each question has a set of at least four possible responses reflecting a range of intensity; for example, "sadness": $0=$ "I do not feel sad"; $1=$ "I feel sad"; $2=$ "I am sad all the time and I can't snap out of it"; 3 = "I am so sad or unhappy that I can't stand it". Higher scores reflect greater severity of depressive symptoms (Cronbach's alpha $=0.88)$.

\section{Statistical analysis}

Of the 129 patients initially enrolled, all but nine completed the study. The statistical analysis was performed as per protocol.

A series of chi-squared tests were computed to check for possible sociodemographic (sex, socioeconomic status, civil status, and job position) differences between the three groups. A one-way analysis of variance was calculated to check for possible age differences. To test whether symptoms of depression and sleep quality differed over time or between 
groups, two analyses of variance for repeated measures were performed with the factors time (pre vs post), group (SHP, LOR, and LOR-SHP), and time by group interaction, and as dependent variables symptoms of depression and sleep quality. Post-hoc tests were performed with Bonferroni-Holm corrections for $P$-values. Effect sizes were reported as partial eta squared $\left(\eta_{\mathrm{p}}^{2}\right)$, and the nominal level of significance was set at $\alpha \leq 0.05$. All statistics was performed with SPSS ${ }^{\circledR} 23.0$ (IBM Corporation, Armonk, NY, USA) for Apple $\mathrm{Mac}^{\circledR}$.

\section{Results}

\section{Psychosocial dimensions of the sample}

Table 1 reports the descriptive and inferential statistics of the sample, separately by the interventions (SHP, LOR, and SHP-LOR).

The three groups did not differ in age, sex distribution, socioeconomic status, civil status, or job position. Accordingly, these dimensions were not introduced as possible confounders.

\section{Sleep disturbances}

Table 2 gives all statistical indices (descriptive and inferential statistics); therefore, statistical indices are not repeated in the text.

As shown in Table 2 and Figure 2, sleep disturbances decreased significantly over time. Neither group differences nor the time by group interaction was significant.

\section{Depression scores}

As shown in Table 2 and Figure 3, symptoms of depression decreased significantly over time. Compared with SHP and LOR-SHP groups, the LOR group had higher depression scores. The significant time by group interaction showed that symptoms of depression decreased significantly more over time in the SHP and SHP-LOR groups than in the LOR group.

\section{Correlations between symptoms of depression and sleep disturbances at the beginning and at the end of the study}

Table 3 gives an overview of the correlations between symptoms of depression and sleep disturbances at the beginning and at the end of the study. While symptoms of depression at the beginning and at the end of the study did significantly correlate, no significant or meaningful correlations were found for sleep disturbances.

\section{Discussion}

The key findings of this first randomized study of three different adjuvant treatment methods to improve sleep and depression among a sample of outpatients with MDD were as follows. All three methods (SHP, LOR, and SHP-LOR) improved sleep, with no advantages for any one method. They improved symptoms of depression, with advantages for the SHP and SHP-LOR conditions; more specifically, the combination (SHP-LOR) reduced symptoms of depression most effectively, a finding, which expands upon the current literature in an important way. Four hypotheses were formulated, and each of these is considered in turn.

Our first hypothesis was that symptoms of depression would reduce over time, and this was fully confirmed. Therefore, the present results confirm the wealth of studies ${ }^{34-37}$ showing a favorable effect of antidepressants on both symptoms of depression and sleep.

Our second hypothesis was that the adjuvant combined treatment (SHP-LOR) would have a more beneficial effect on sleep than the other two treatment methods (SHP and LOR), but this was not confirmed; all three interventions had comparable effects. Therefore, the pattern of results is at odds with previous research. ${ }^{26-28}$ The pattern of results suggests that treatment with adjuvant LOR and with adjuvant psychoeducation had similarly beneficial effects on sleep. However, given the possible risk of benzodiazepine dependence, ${ }^{52,53}$

Table I Descriptive and inferential statistics of sociodemographic variables, separately for the three study conditions (SHP, LOR, and SHP-LOR)

\begin{tabular}{|c|c|c|c|c|}
\hline \multirow[t]{2}{*}{ Sociodemographic variables } & \multicolumn{3}{|l|}{ Groups } & \multirow[t]{2}{*}{ Statistics } \\
\hline & SHP & LOR & SHP-LOR & \\
\hline $\mathrm{n}$ & 40 & 40 & 40 & \\
\hline $\operatorname{Sex}(f / m)$ & $23 / 17$ & $21 / 19$ & $34 / 16$ & $\chi^{2}(\mathrm{~N}=120, d f=2)=0.48, P>0.1$ \\
\hline Age (years) & $46.88(8.60)$ & $49.23(7.92)$ & $48.65(8.73)$ & $F(2, I I 7)=I .43, P>0 . I$ \\
\hline Socioeconomic status (low/middle/high) & $26 / 14 / 0$ & $33 / 7 / 0$ & $27 / 13 / 0$ & $\chi^{2}(\mathrm{~N}=120, d f=2)=3.53, P>0.1$ \\
\hline Civil status (single/married/single-separated) & $2 / 36 / 2$ & $4 / 35 / 1$ & $5 / 31 / 4$ & $\chi^{2}(\mathrm{~N}=120, d f=4)=3.68, P>0.1$ \\
\hline Job position (employed/housework/unemployed) & $27 / 5 / 8$ & $24 / 3 / 13$ & $23 / 7 / 10$ & $\chi^{2}(\mathrm{~N}=120, d f=4)=3.18, P>0.1$ \\
\hline
\end{tabular}

Abbreviations: SHP, sleep hygiene program; LOR, lorazepam; f/m, female/male; SHP-LOR, sleep hygiene program combined with lorazepam. 


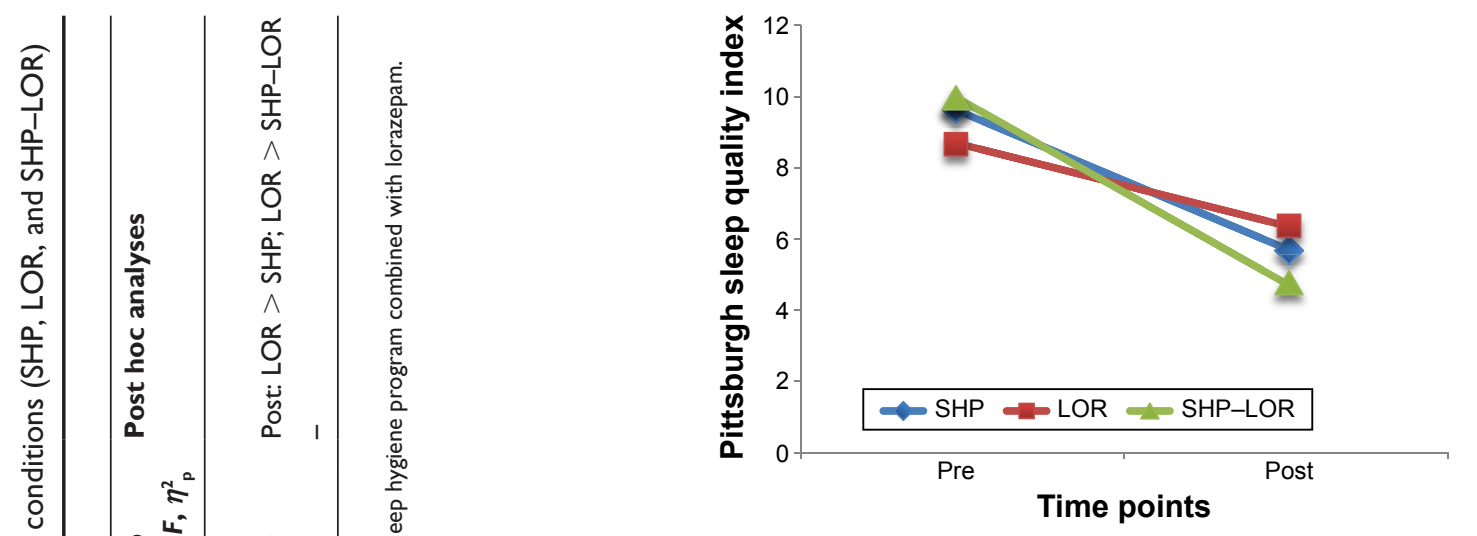

Figure 2 Sleep disturbances significantly decreased over time irrespective of intervention.

Note: Points are mean values.

Abbreviations: SHP, sleep hygiene program; LOR, lorazepam; SHP-LOR, sleep hygiene program combined with lorazepam.

clinicians should balance the risks and benefits of LOR or benzodiazepine prescription against psychoeducational interventions; these latter in the short term need more engagement to acquire the necessary skills but in the long run have no side effects and improve patients' self-efficacy.

To explain why psychoeducational interventions may work, theoretical models suggest that knowledge alone is rarely sufficient to achieve behavioral change. Rather, according to social cognitive models such as the theory of planned behavior, behavior is influenced by intentions and perceived control of behavior and the importance accorded to the intervention by significant others. To put it another way, the determining factors are whether the recipients of sleep education perceive the behavior to be desirable, positive, or worthwhile and the degree to which others, such as relatives, friends, or colleagues, approve of healthy sleep behavior..$^{54-56}$

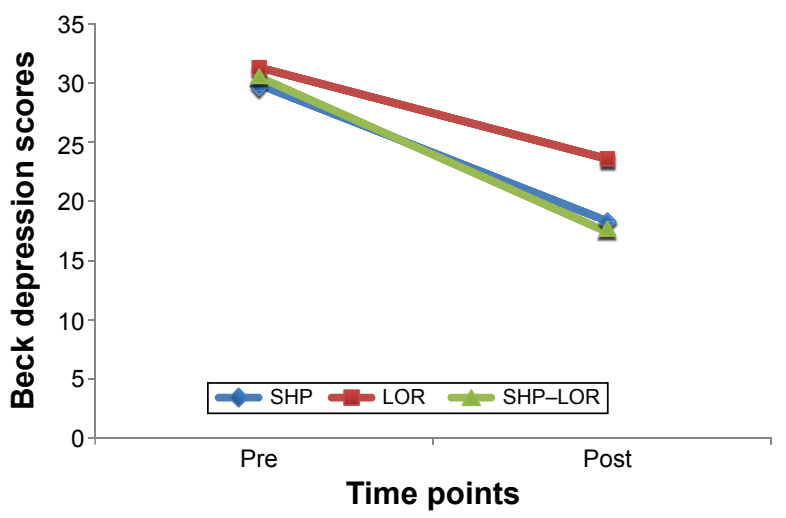

Figure 3 Symptoms of depression decreased significantly over time in all three interventions.

Notes: Compared with the LOR condition, the SHP and the SHP-LOR had superior effects on symptoms of depression. Points are means.

Abbreviations: SHP, sleep hygiene program; LOR, lorazepam; SHP-LOR, sleep hygiene program combined with lorazepam. 
Table 3 Overview of the correlations (Pearson's $r$ ) between symptoms of depression and sleep disturbances at the beginning and at the end of the study

\begin{tabular}{llllll}
\hline & BDI and & Groups & & \\
\cline { 3 - 6 } & PSQI & $\mathbf{I , r}$ & $\mathbf{2 , r}$ & $\mathbf{3 , r}$ & $\mathbf{4 , \boldsymbol { r }}$ \\
\hline $\mathrm{I}$ & BDI pre & - & $0.25^{*}$ & -0.04 & 0.17 \\
2 & BDI post & & - & -0.06 & 0.07 \\
3 & PSQI pre & & & - & -0.03 \\
4 & PSQI post & & & & - \\
\hline Note: & $*$ P $<0.05$. \\
Abbreviations: BDI, Beck Depression Inventory; PSQI, Pittsburgh Sleep Quality \\
Index.
\end{tabular}

Our third hypothesis was that the adjuvant combined treatment (SHP-LOR) would have a more beneficial effect on depression than the other two treatment methods (SHP and LOR), and this was confirmed; this combination of treatments led to a greater reduction in symptoms of depression, though the SHP alone also produced greater improvement than the intervention with LOR which had the smallest impact. These findings, therefore, accord with previous research. ${ }^{31,32,57,58}$ In light of these findings, and if we again consider the potential risk of benzodiazepine dependence, ${ }^{52,53}$ clinicians should balance the risks and benefits of LOR or benzodiazepine prescription. This caution is particularly relevant given that psychotherapeutic and psychoeducational programs usually have a long-lasting effect for a broad variety of mental illnesses,${ }^{59}$ depression, ${ }^{60-63}$ and sleep problems. ${ }^{64-68}$

Our fourth hypothesis was that symptoms of depression and sleep disturbances would be associated both at the beginning and at the end of the study, but the results did not support this. Rather, the results pattern suggests that symptoms of depression and sleep disturbances were not connected. This finding demands particular attention given that symptoms of depression and sleep disturbances are usually highly intertwined. ${ }^{10,38-40}$ Specifically, Fava ${ }^{41}$ reported that insomnia and daytime sleepiness were often associated with depression. Strikingly, Lovato and Gradisar ${ }^{14}$ showed in their metaanalysis that, among adolescents, sleep disturbances preceded symptoms of depression and not vice versa. The evidence available from the present study cannot shed any light on the underlying psychological and physiological mechanisms. However, we offer the following speculative interpretations. First, it is conceivable that the tool (PSQI ${ }^{47}$ ) was too coarse grained to detect subtle changes in sleep. Responses restricted to a four-point Likert scale might be too insensitive to register changes. On the other hand, the PSQI is an internationally well-regarded and validated instrument in sleep research. Second, floor effects are possible given that one item asks for intake of sleep medication, and patients were bound to score high because they were under antidepressant treatment and in case of the LOR group, under additional benzodiazepine. Third, there was really no higher improvement.

The novelty of the findings should be balanced against the following limitations. First, participants were not blind as to treatment, and therefore, it is conceivable that expectancies and motivational processes might have biased the results. Second, it is also conceivable that further latent but unassessed psychophysiological factors might have biased two or more variables in the same or opposite direction. Third, evidence at the neuronal and endocrinological levels such as cortisol, growth hormone, alpha-amylase, or BDNF could have shed more light on the neurophysiological processes underlying the positive changes in symptoms of depression and sleep disturbances. Fourth, objective sleep measurements (actigraphy and sleep-EEG) would have been helpful in estimating objective changes in sleep. However, for larger samples such as the present one, questionnaires are still the gold standard. ${ }^{69}$ Fifth, we relied on self-ratings. Future studies might also collect experts' ratings as regards patients' symptoms of depression. Specifically, experts could have rated patients' symptoms of depression with the Montgomery-Asberg Depression Rating Scale, which consists of ten items and has been recently validated. ${ }^{70}$ Sixth, we assessed neither the intensity nor the quality of the patienttherapist time. Though patients in the LOR condition had four pharmacotherapy sessions, it is conceivable that patients in the sleep hygiene psychoeducation had further benefits from the psychotherapeutic setting. Seventh, we performed a per protocol analysis, whereas an intention-to-treat approach would have been more correct; however, comparing the pattern of results of the two statistical approaches, no descriptive or significant differences were observed. Last, it would have been very useful and important to follow up these patients, as there is some, but as yet insufficient, evidence that psychotherapeutic interventions have a long-lasting effect than psychopharmacotherapy. ${ }^{29}$

\section{Conclusion}

The findings of this study indicate that an adjuvant SHP and its combination with LOR can produce greater improvements in symptoms of depression. As regards to sleep disturbances, no intervention (SHP, LOR, and SHP-LOR) showed superior effects. Therefore, in light of the increased risk of benzodiazepine dependence, clinicians should carefully balance the risks and benefits in prescribing LOR as an add-on to treat both depressive disorders and sleep disturbances. 


\section{Acknowledgments}

The authors thank Nick Emler (University of Surrey, UK) for proofreading the manuscript. The work of the Farshchian Psychiatry Hospital was financially supported by Research Center for Behavioral Disorders and Substances Abuse, Hamadan University of Medical Sciences (grant number 903222865).

\section{Disclosure}

The authors report no conflicts of interest in this work.

\section{References}

1. Bayon V, Leger D, Gomez-Merino D, Vecchierini MF, Chennaoui M. Sleep debt and obesity. Ann Med. 2014;46(5):264-272.

2. Brand S, Kirov R, Kalak N, et al. Poor sleep is related to lower emotional competence among adolescents. Behav Sleep Med. 2015:1-13.

3. Franzen PL, Buysse DJ, Dahl RE, Thompson W, Siegle GJ. Sleep deprivation alters pupillary reactivity to emotional stimuli in healthy young adults. Biol Psychol. 2009;80(3):300-305.

4. Genzel L, Ahrberg K, Roselli C, et al. Sleep timing is more important than sleep length or quality for medical school performance. Chronobiol Int. 2013;30(6):766-771.

5. Curcio G, Ferrara M, De Gennaro L. Sleep loss, learning capacity and academic performance. Sleep Med Rev. 2006;10(5):323-337.

6. Hunter P. Sophisticated sleep improves our brains: our advanced cognitive and social skills might derive from the evolution of improved sleep quality; today, sleep therapy could help with mental health issues and learning. EMBO Rep. 2016;17(3):296-299.

7. Nutt D, Wilson S, Paterson L. Sleep disorders as core symptoms of depression. Dialogues Clin Neurosci. 2008;10(3):329-336.

8. Buysse DJ, Cheng Y, Germain A, et al. Night-to-night sleep variability in older adults with and without chronic insomnia. Sleep Med. 2010;11(1): 56-64.

9. Srinivasan V, Pandi-Perumal SR, Trakht I, et al. Pathophysiology of depression: role of sleep and the melatonergic system. Psychiatry Res. 2009;165(3):201-214.

10. Hatzinger M, Hemmeter UM, Brand S, Ising M, Holsboer-Trachsler E. Electroencephalographic sleep profiles in treatment course and longterm outcome of major depression: association with DEX/CRH-test response. J Psychiatr Res. 2004;38(5):453-465.

11. Menza M, Marin H, Opper RS. Residual symptoms in depression: can treatment be symptom-specific? J Clin Psychiatry. 2003;64(5): 516-523.

12. Taylor DJ, Lichstein KL, Durrence HH, Reidel BW, Bush AJ. Epidemiology of insomnia, depression, and anxiety. Sleep. 2005;28(11): 1457-1464.

13. van Mill JG, Hoogendijk WJ, Vogelzangs N, van Dyck R, Penninx BW. Insomnia and sleep duration in a large cohort of patients with major depressive disorder and anxiety disorders. J Clin Psychiatry. 2010; 71(3):239-246.

14. Lovato N, Gradisar M. A meta-analysis and model of the relationship between sleep and depression in adolescents: recommendations for future research and clinical practice. Sleep Med Rev. 2014;18(6):521-529.

15. Plante DT, Winkelman JW. Sleep disturbance in bipolar disorder: therapeutic implications. Am J Psychiatry. 2008;165(7):830-843.

16. Breslau N, Roth T, Rosenthal L, Andreski P. Sleep disturbance and psychiatric disorders: a longitudinal epidemiological study of young adults. Biol Psychiatry. 1996;39(6):411-418.

17. Krakow B, Ribeiro JD, Ulibarri VA, Krakow J, Joiner TE Jr. Sleep disturbances and suicidal ideation in sleep medical center patients. J Affect Disord. 2011;131(1-3):422-427.
18. Rumble ME, White KH, Benca RM. Sleep disturbances in mood disorders. Psychiatr Clin North Am. 2015;38(4):743-759.

19. Buysse DJ, Tu XM, Cherry CR, et al. Pretreatment REM sleep and subjective sleep quality distinguish depressed psychotherapy remitters and nonremitters. Biol Psychiatry. 1999;45(2):205-213.

20. Bjørngaard JH, Bjerkeset O, Romundstad P, Gunnell D. Sleeping problems and suicide in 75,000 Norwegian adults: a 20 year follow-up of the HUNT I study. Sleep. 2011;34(9):1155-1159.

21. Gangwisch JE, Babiss LA, Malaspina D, Turner JB, Zammit GK, Posner K. Earlier parental set bedtimes as a protective factor against depression and suicidal ideation. Sleep. 2010;33(1):97-106.

22. Fava M, Asnis GM, Shrivastava RK, et al. Improved insomnia symptoms and sleep-related next-day functioning in patients with comorbid major depressive disorder and insomnia following concomitant zolpidem extended-release $12.5 \mathrm{mg}$ and escitalopram treatment: a randomized controlled trial. J Clin Psychiatry. 2011;72(7):914-928.

23. Asnis GM, Thomas M, Henderson MA. Pharmacotherapy treatment options for insomnia: a primer for clinicians. Int $J \mathrm{Mol} \mathrm{Sci}$. 2016;17:50.

24. Asnis GM, Chakraburtty A, DuBoff EA, et al. Zolpidem for persistent insomnia in SSRI-treated depressed patients. J Clin Psychiatry. 1999; 60(10):668-676.

25. Fava M, Asnis GM, Shrivastava R, et al. Zolpidem extended-release improves sleep and next-day symptoms in comorbid insomnia and generalized anxiety disorder. J Clin Psychopharmacol. 2009;29(3): 222-230.

26. Zachariae R, Lyby MS, Ritterband LM, O'Toole MS. Efficacy of internet-delivered cognitive-behavioral therapy for insomnia - a systematic review and meta-analysis of randomized controlled trials. Sleep Med Rev. 2015;30:1-10.

27. Tal JZ, Suh SA, Dowdle CL, Nowakowski S. Treatment of insomnia, insomnia symptoms, and obstructive sleep apnea during and after menopause: therapeutic approaches. Curr Psychiatry Rev. 2015;11(1): 63-83.

28. Haynes P. Application of cognitive behavioral therapies for comorbid insomnia and depression. Sleep Med Clin. 2015;10(1):77-84.

29. Mitchell MD, Gehrman P, Perlis M, Umscheid CA. Comparative effectiveness of cognitive behavioral therapy for insomnia: a systematic review. BMC Fam Pract. 2012;13:40.

30. Riemann D, Perlis ML. The treatments of chronic insomnia: a review of benzodiazepine receptor agonists and psychological and behavioral therapies. Sleep Med Rev. 2009;13(3):205-214.

31. Manber R, Kraemer HC, Arnow BA, et al. Faster remission of chronic depression with combined psychotherapy and medication than with each therapy alone. J Consult Clin Psychol. 2008;76(3):459-467.

32. Manber R, Edinger JD, Gress JL, San Pedro-Salcedo MG, Kuo TF, Kalista T. Cognitive behavioral therapy for insomnia enhances depression outcome in patients with comorbid major depressive disorder and insomnia. Sleep. 2008;31(4):489-495.

33. Manber R, Chambers AS. Insomnia and depression: a multifaceted interplay. Curr Psychiatry Rep. 2009;11(6):437-442.

34. Amick HR, Gartlehner G, Gaynes BN, et al. Comparative benefits and harms of second generation antidepressants and cognitive behavioral therapies in initial treatment of major depressive disorder: systematic review and meta-analysis. BMJ. 2015;351:h6019.

35. Santarsieri D, Schwartz TL. Antidepressant efficacy and sideeffect burden: a quick guide for clinicians. Drugs Context. 2015;4: 212290.

36. Tundo A, de Filippis R, Proietti L. Pharmacologic approaches to treatment resistant depression: evidences and personal experience. World J Psychiatry. 2015;5(3):330-341.

37. Zhou X, Qin B, Whittington C, et al. Comparative efficacy and tolerability of first-generation and newer-generation antidepressant medications for depressive disorders in children and adolescents: study protocol for a systematic review and network meta-analysis. BMJOpen. 2015;5(9):e007768. 
38. Steiger A, Kimura M. Wake and sleep EEG provide biomarkers in depression. J Psychiatr Res. 2010;44(4):242-252.

39. Steiger A, Dresler M, Kluge M, Schüssler P. Pathology of sleep, hormones and depression. Pharmacopsychiatry. 2013;46(suppl 1): S30-S35.

40. Hori H, Koga N, Hidese S, et al. 24-h activity rhythm and sleep in depressed outpatients. J Psychiatr Res. 2016;77:27-34.

41. Fava M. Daytime sleepiness and insomnia as correlates of depression. J Clin Psychiatry. 2004;65(suppl 16):27-32.

42. Sheehan DV, Lecrubier Y, Sheehan KH, et al. The Mini-International Neuropsychiatric Interview (M.I.N.I.): the development and validation of a structured diagnostic psychiatric interview for DSM-IV and ICD-10. J Clin Psychiatry. 1998;59(suppl 20):22-33. quiz 34-57.

43. Bastien $\mathrm{CH}$, Vallières $\mathrm{A}$, Morin $\mathrm{CM}$. Validation of the Insomnia Severity Index as an outcome measure for insomnia research. Sleep Med. 2001;2(4):297-307.

44. Silberman SA. The Insomnia Workbook. Oakland, CA: New Harbinger Publications, Inc; 2008.

45. Brand S, Kalak N, Gerber M, Kirov R, Pühse U, Holsboer-Trachsler E. High self-perceived exercise exertion before bedtime is associated with greater objectively assessed sleep efficiency. Sleep Med. 2014; 15(9):1031-1036.

46. Nazifi M, Mokarami H, Akbaritabar A, Kalte O, Rahi A. Psychometric properties of the Persian translation of Pittsburgh Sleep Quality Index. Health Scope. 2014;3(2):e15547.

47. Buysse DJ, Reynolds CF 3rd, Monk TH, Berman SR, Kupfer DJ. The Pittsburgh Sleep Quality Index: a new instrument for psychiatric practice and research. Psychiatry Res. 1989;28(2):193-213.

48. Knutson KL, Rathouz PJ, Yan LL, Liu K, Lauderdale DS. Stability of the Pittsburgh Sleep Quality Index and the Epworth Sleepiness Questionnaires over 1 year in early middle-aged adults: the CARDIA study. Sleep. 2006;29(11):1503-1506.

49. Carpenter JS, Andrykowski MA. Psychometric evaluation of the Pittsburgh Sleep Quality Index. J Psychosom Res. 1998;45(1):5-13.

50. Beck AT, Ward CH, Mendelson M, Mock J, Erbaugh J. An inventory for measuring depression. Arch Gen Psychiatry. 1961;4:561-571.

51. Ghassemzadeh H, Mojtabai R, Karamghadiri N, Ebrahimkhani N. Psychometric properties of a Persian-language version of the Beck Depression Inventory - Second edition: BDI-II-PERSIAN. Depress Anxiety. 2005;21(4):185-192.

52. Chouinard G. Issues in the clinical use of benzodiazepines: potency, withdrawal, and rebound. J Clin Psychiatry. 2004;65(suppl 5):7-12.

53. Rosenberg RP. Sleep maintenance insomnia: strengths and weaknesses of current pharmacologic therapies. Ann Clin Psychiatry. 2006;18(1): 49-56.

54. Harvey AG. Pre-sleep cognitive activity: a comparison of sleep-onset insomniacs and good sleepers. Br J Clin Psychol. 2000;39(pt 3): 275-286.

55. Harvey AG. Sleep hygiene and sleep-onset insomnia. J Nerv Ment Dis. 2000;188(1):53-55.
56. Espie CA, Inglis SJ, Harvey L, Tessier S. Insomniacs' attributions. Psychometric properties of the Dysfunctional Beliefs and Attitudes about Sleep Scale and the Sleep Disturbance Questionnaire. J Psychosom Res. 2000;48(2):141-148.

57. Ong JC, Shapiro SL, Manber R. Combining mindfulness meditation with cognitive-behavior therapy for insomnia: a treatment-development study. Behav Ther. 2008;39(2):171-182.

58. Morin CM, Vallières A, Guay B, et al. Cognitive behavioral therapy, singly and combined with medication, for persistent insomnia: a randomized controlled trial. JAMA. 2009;301(19):2005-2015.

59. Zhao S, Sampson S, Xia J, Jayaram MB. Psychoeducation (brief) for people with serious mental illness. Cochrane Database Syst Rev. 2015;4: CD010823.

60. Aki A, Tomotake M. Changes of levels of depression and quality of life after short-term cognitive behavioral educational program for adolescent students in health class. J Med Invest. 2015;62(3-4):204-208.

61. Casanas R, Catalan R, Penades R, et al. Evaluation of the effectiveness of a psychoeducational intervention in treatment-naive patients with antidepressant medication in primary care: a randomized controlled trial. ScientificWorldJournal. 2015;2015:718607.

62. Linde K, Rücker G, Sigterman K, et al. Comparative effectiveness of psychological treatments for depressive disorders in primary care: network meta-analysis. BMC Fam Pract. 2015;16:103.

63. Tanaka S, Ishikawa E, Mochida A, Kawano K, Kobayashi M. Effects of early-stage group psychoeducation programme for patients with depression. Occup Ther Int. 2015;22(4):195-205.

64. de Bruin EJ, Bögels SM, Oort FJ, Meijer AM. Efficacy of cognitive behavioral therapy for insomnia in adolescents: a randomized controlled trial with internet therapy, group therapy and a waiting list condition. Sleep. 2015;38(12):1913-1926.

65. Harvey AG, Soehner AM, Kaplan KA, et al. Treating insomnia improves mood state, sleep, and functioning in bipolar disorder: a pilot randomized controlled trial. J Consult Clin Psychol. 2015;83(3):564-577.

66. Kloss JD, Nash CO, Walsh CM, Culnan E, Horsey S, Sexton-Radek K. A "Sleep 101" program for college students improves sleep hygiene knowledge and reduces maladaptive beliefs about sleep. Behav Med. 2016;42(1):48-56.

67. Sadler P, McLaren S, Klein B, Jenkins M, Harvey J. Cognitive behaviour therapy for older adults experiencing insomnia and depression in a community mental health setting: study protocol for a randomised controlled trial. Trials. 2015;16:538.

68. Ong JC, Shapiro SL, Manber R. Mindfulness meditation and cognitive behavioral therapy for insomnia: a naturalistic 12-month follow-up Explore (NY). 2009;5(1):30-36.

69. Wolfson AR, Carskadon MA, Acebo C, et al. Evidence for the validity of a sleep habits survey for adolescents. Sleep. 2003;26(2):213-216.

70. Ahmadpanah M, Sheikhbabaei M, Haghighi M, et al. Validity and test-retest reliability of the Persian version of the MontgomeryAsberg Depression Rating Scale. Neuropsychiatr Dis Treat. 2016;12: 603-607.
Neuropsychiatric Disease and Treatment

\section{Publish your work in this journal}

Neuropsychiatric Disease and Treatment is an international, peerreviewed journal of clinical therapeutics and pharmacology focusing on concise rapid reporting of clinical or pre-clinical studies on a range of neuropsychiatric and neurological disorders. This journa is indexed on PubMed Central, the 'PsycINFO' database and CAS,

\section{Dovepress}

and is the official journal of The International Neuropsychiatric Association (INA). The manuscript management system is completely online and includes a very quick and fair peer-review system, which is all easy to use. Visit http://www.dovepress.com/testimonials.php to read real quotes from published authors. 